Im Journal Club referieren und kommentieren diese Experten für Sie Arbeiten aus der internationalen Fachliteratur.

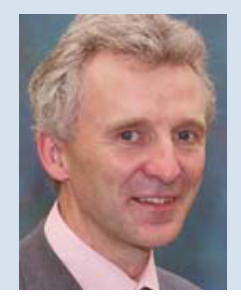

Dr. med.

Hartmut

Koch,

Vechta

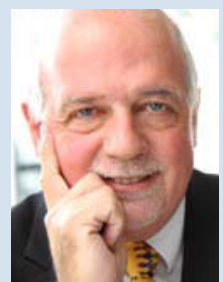

Prof. Dr. med. Jens C. Möller, Saarbrücken

\section{Ursachenforschung bei Schreikindern}

\section{Exzessives Schreien kann ein Hinweis auf ein unerkanntes organisches Problem sein. Deshalb machte sich ein US-amerikanisches Team auf die Suche nach einem möglichen Zusammenhang zwischen Darminflam- mation und Säuglingskoliken.}

$\mathrm{D}$ ie Suche nach einer organischen Ursache von funktionellen Bauchschmerzen ist mit den üblichen Methoden nicht zielführend. Marc Rhoads und Kollegen aus Houston, Texas, sind deshalb der Frage nachgegangen, ob nicht doch entzündliche Vorgänge und zusätzlich noch eine geänderte Kolonflora maßgebend sein könnten. Sie untersuchten 36 „Schreikinder“ im Alter von zwei Wochen bis drei Monaten. Die Schreikinder hatten im Durchschnitt $314 \pm 36$ Minuten pro Tag geschrien, die 17 Kontrollkinder $103 \pm 17$ Minuten. Gemessen wurden das fäkale Calprotectin (Marker für neutrophile Infiltration), Mikroorganismen (Gradienten-Gelelektrophorese, Klonierung und Sequenzierung) und $\mathrm{H}_{2}$ Exhalation.

Als Ergebnis zeigte sich, dass das fäkale Calprotectin bei den Schreikindern zweimal so hoch war wie das der Kontrollkinder. Klebsiella-Spezies waren deutlich häufiger bei den Schreikindern, während Enterobacter- bzw. Pantoea-Spezies nur bei den Kontrollkindern beobachtet wurden. Bei der Messung von Wasserstoffexhalation bei nüchternene Säuglingen und nach Nahrungsaufnahme fanden sich keine signifikanten Differenzen, wobei ein Trend zu höheren $\mathrm{H}_{2}$-Konzentrationen in der Kolikgruppe vor allem postprandial auffiel.

Die Autoren schließen daraus, dass die sogenannten funktionellen Bauch- schmerzen bei Säuglingen eine entzündliche Komponente haben und die Beschwerden mit einer veränderten Darmflora in Zusammenhang stehen. Sie möchten daher eine Interventionsstudie mit einem Probiotikum ins Leben rufen.

Kommentar: Diese Studie mit einer relativ kleinen Zahl von Patienten (ein Selektionsbias ist nicht auszuschließen!) kann zunächst als Pilotstudie gewertet werden, sollte aber Anlass zu weiteren Forschungen in diese Richtung geben.

Die Erhöhung der Calprotectinkonzentration im Stuhl spiegelt eine Neutrophilenaktivierung in der Mukosa des Gastrointestinaltraktes wider. Die Ursache hierfür lässt sich allerdings nicht differenzieren. Es kommen sowohl bakeriell bedingte als auch allergische Entzündungen in Frage. Das Calprotectin hat gegenüber dem auch in Deutschland gerne angewendeten Lactoferrin den Vorteil, dass es bei gestillten Kindern durch Muttermilch nicht gestört wird. Man kann über die Ursache entzündlicher Veränderungen in der Gruppe der Kinder mit Koliken zunächst nur spekulieren. Frühere Studien konnten zeigen, dass bei einem Teil der Kinder eine Kuhmilchunverträglichkeit vorliegt.

Auch der Trend zu höheren postprandialen Wasserstoffexhalationen können ein Hinweis auf veränderte Bedingungen im kindlichen Gastrointestinaltrakt sein.
Interessant ist vor allem die neue Methode der denaturierenden Gradienten-Gelelektrophorese der intestinalen Flora. Diese Methode kann 16S-rRNA-Gensegmente der intestinalen Bakterienflora nachweisen. Die Untersuchung hat den großen Vorteil, dass sie auch abgestorbene Bakterien erfasst. Sie wird in $\mathrm{Zu}-$ kunft völlig neue Möglichkeiten der Analyse der Stuhlflora bieten und auch bei anderen Krankheiten angewendet werden. Gegenüber der in Deutschland noch gerne von kommerziellen Labors angebotenen Stuhlfloraanalyse durch Kultivierung von Stuhlproben hat sie den Vorteil, nicht durch den Einfluss von Sauerstoff auf die zu 90\% anaerobe Flora des Darms gestört zu werden. Die Ergebnisse der Gelelektrophorese bei den Kindern mit Koliken sprechen jedenfalls für signifikante Unterschiede der Bakterienzusammensetzung.

Die Untersuchung bietet neue Ansatzpunkte zu weiteren Forschungen, die sicher aber noch mehrere Jahre in Anspruch nehmen werden. In der Zwischenzeit gilt weiterhin die Regel für alle Pädiater, dass exzessives Schreien nicht nur ein Hinweis auf ein unerkanntes organisches Problem sein kann, sondern auch auf eine gestörte Eltern-Kind-Interaktion deuten kann. Die Studie zeigt aber auch, dass für die Betreuung von schreienden Säuglingen nicht nur die mit Psychotherapeuten besetzte Schreiambulanz zuständig sein sollte, sondern auch der Kinderarzt mit seiner pädiatrischgastroenterologischen Erfahrung.

\section{Dr. Hartmut Koch/ Dr. Martin Claßen}

Rhoads JM et al. Altered microflora and increased fecal calprotectin in infants with colic. J Pediatr 2009; 155: 823-8 